\title{
A Short-Cut Design Technique to Batch Distillation Column
}

\author{
Wenfeng Hao ${ }^{1,2,3,4}$ \\ ${ }^{1}$ Department of Applied Chemistry, School of Renewable Energy, Shenyang Institute of Engineering, Shenyang, China \\ ${ }^{2}$ Liaoning Key Laboratory of Chemical Separation Technology, College of Chemical Engineering, Shenyang University of \\ Chemical Technology, Shenyang, China \\ ${ }^{3}$ Center for Natural Gas Hydrate Research, CAS Key Laboratory of Gas Hydrate, Guangzhou Institute of Energy Conversion, \\ Chinese Academy of Sciences, Guangzhou, China \\ ${ }^{4}$ Institute of Adsorption and Inorganic Membrane, State Key Laboratory of Fine Chemicals, Dalian University of Technology, \\ Dalian, China \\ Email: haowenfeng@163.com
}

How to cite this paper: Hao, W.F. (2019) A Short-Cut Design Technique to Batch Distillation Column. Advances in Chemical Engineering and Science, 9, 263-279. https://doi.org/10.4236/aces.2019.93020

Received: May 5, 2019

Accepted: July 28, 2019

Published: July 31, 2019

Copyright (C) 2019 by author(s) and Scientific Research Publishing Inc. This work is licensed under the Creative Commons Attribution International License (CC BY 4.0).

http://creativecommons.org/licenses/by/4.0/ (c) (i) Open Access

\begin{abstract}
The effects of equipment parameters of batch distillation column on the yield proportion are discussed and analyzed, the relations between maximal yield proportion and the column equipment parameters are correlated, which not only can be used to appraise rationality of the design parameters of the columns being employed and which but also can be used to new batch distillation column design. Under the assistance of the separation difficulty defined in this paper, the minimum number of theoretical plates is determined by the limit loss proportion method given, and further the actual number of theoretical plates and the height for the batch distillation are calculated by using the redundancy coefficient found to complete the whole design of the batch distillation as shown in the computational sample. Research showed that the actual number of theoretical plates and the height of batch distillation column with the column diameter $0.6 \mathrm{~m}$ are 17 and $5.1 \mathrm{~m}$ in alcohol mixture separation system of the sample proposed. Moreover, the approach can be extended to the design of batch distillation column with a separation system of multi-component liquid mixture after those adjacent components are treated as numerous binary component systems.
\end{abstract}

\section{Keywords}

Design Technique, Batch Distillation Column, Separation Difficulty, Redundancy Coefficient, Theoretical Plate

\section{Introduction}

Batch distillation as a significant and flexible separation approach is frequently 
employed in pharmaceutical, food, and petrochemical industries to acquire manifold high value-added and high-purity products. Generally batch distillation research can be divided into two classifications whereby they are operational type and design type. On the one hand, as a significant research branch, operational problems mainly involve modelling, simulation, optimization, control and so on [1] [2] [3] [4]. Batch distillation columns with different structures were being used to implement operational investigation according to their separation tasks with different requirements.

A new operation mode for reactive batch distillation in middle-vessel columns was proposed, depending upon the characteristics of the reaction mixture [5]. Multi-objective dynamic optimizations of novel batch distillation utilizing an evolutionary algorithm were presented and the obtained results showed the feasibility of the proposed methodology for the dynamic process in a middle vessel batch distillation [6]. The control structures of conventional middle-vessel batch distillation and modified middle-vessel batch distillation were compared, and thus the results were that level controllers could have obtained high purity products when the feed composition was kept constant [7]. The control structures of middle vessel batch distillation for separating a ternary system were investigated, and the results showed that a composition control structure and temperature control structure could perform well for controlling the product purity and liquid holdup [8]. The composition control structure and double temperature control structures were compared and the results indicated that the temperature control structures performed better were compared [9]. The mixture of dimethyl carbonate, ethyl methyl carbonate and diethyl carbonate was separated by middle-vessel batch distillation with feeding in middle-vessel and good control effects were achieved [10]. A novel operation policy for the separation of zeotropic mixtures via batch distillation column with a side withdrawal was examined and comparison results to batch distillation column with a middle vessel simultaneously were given [11]. The cyclic operation of a middle vessel batch distillation had been carried out in theoretical and experimental investigations and synchronously the operating behaviors in the distillation configuration were described using a mathematical model [12]. Optimization to reboiler duty and reflux ratio profiles of vapor recompressed batch distillation were implemented and a cluster of optimum points formally known as the Pareto-optimal front were found, resulting in one optimal point that was selected utilizing the technique for order of preference [13]. Moreover, batch distillation control as another direction also was widely investigated to ensure stable operational behavior. Using proposed Luenberger-like nonlinear estimator (LNE) and the feedback linearizing controller (FLC) the discrepancy of process/model mismatch was efficiently corrected [14]. A technique for nonlinear system identification and model reduction using artificial neural networks was proposed and was succeeded in applying for batch distillation control [15]. Fuzzy algorithms in batch distillation column control were employed, reducing the batch time and the energy consumption [16]. Simultaneous optimization of equipment design 
and process operation for batch reactive distillation processes were investigated to develop an efficient algorithm, considering the effect of design and operating variables on total annual cost [17].

Although all of above references showed achievements in operational aspects of batch distillation, investigators' contributions to design type in batch distillation also circumvent touched problems within their research stages. A short-cut method as a replacement of simulated continuous distillation column design channels was mentioned and provided reasonably and effectively accurate solutions in designing batch distillation columns, evading much iterative procedures and considerable computational effort in rigorous models [18]. Modified algorithm may succeed in applying for the design of binary, azeotropic batch distillation columns [19]. The batch sizes, operating times, equipment sizes, utility loads, and for the batch distillation of non-ideal multi-component mixtures were estimated using their proposed methods [20]. Short-cut procedure for the design of batch distillations which was equivalent to the Fenske-Underwood-Gilliland procedure for continuous distillations was presented which was used to compute the minimum number of stages and minimum reflux ratio required for batch distillation [21]. A mathematical programming approach to the optimal design of batch distillation columns was given and capital and operating cost contribution also were taken into account. Consequently the column design parameters and operating policy were determined [22]. The optimal design of multi-vessel batch distillation based on an economics performance index was carried out by adopting an evolutionary adaptive search technique. The combined energy efficiency and production rate of the multi-vessel system was found to be greater than that of the regular column, and the benefit was more prominent when separating mixtures with more components [23]. A simplified methodology for the analysis and design of reactive batch distillation columns based on the McCabe-Thiele method for reactive continuous columns and on the concept of a reactive difference point was proposed [24]. The conceptual approach to design the recovery of 2-propanol from a mixture containing water by using cyclohexane as an entrainer under the assistance of computer-aided software tool was done [25]. A short-cut method for batch distillation columns working at constant reflux was applied to solve a problem of four components that needed to be separated and purified to a mole fraction of 0.97 or better [26]. Membrane distillation with feed recirculation for high recovery brine concentration was implemented for the design and operation, whose obtained results had better value for application [27].

Abovementioned fruits regarding design types in batch distillation were better promotors whether in a generalized level or in an idiographic case, but their computations still were complex or were merely applicable to specific circumstances. As a result aim of this investigation is to propose a shortcut design technique to determine the theoretical and actual plate number of the batch distillation column and the height of the batch distillation column to solve the com- 
plexity problems that the batch distillation column was designed by referring to the continuous distillation column design theories or to overcome the uncertainty shortcomings that the design of the batch distillation column originated from the empirical method and the semi-empirical method and further set an example to lead the way that can guide the design of batch distillation column from binary system separation to multi-component system separation.

\section{Equipment Parameters of Batch Distillation Column and Limit Loss Proportion}

\subsection{Discussion to Equipment Parameters of Batch Distillation Column}

It is highly important for evaluating the rationality of the design of batch distillation column to study the effects of the established separation task and of the equipment parameters on the component yield of separation process in a batch distillation column. The effects of operating parameters such as reflux ratio, operating pressure and operating steam velocity on product recovery yield have been well known [28]. Therefore, in a binary separation system some main equipment parameters that affect the separation results will be discussed in this section to pave the way for the succeeding study.

\subsubsection{Number of the Theoretical Plate}

A certain number of theoretical plates are the guarantee to complete the separation task. Generally too few theoretical plate number not only can't make the light component achieve a fixed concentration multiple like a continuous distillation, but also it can't reach the concentration requirements. Moreover, too many theoretical plates have little effect on improving the light component recovery yield, and thus the investment in equipment will be greatly increased. Figure 1 is a schematic diagram of the effects of the theoretical plate on the product yield in a batch distillation with a binary component separation system.

As shown curve $\varphi_{1}$ in Figure 1 the recovery yield of light component at column top is greatly affected and it is not able to satisfy the requirement of the separation when the number of theoretical plates is smaller. With the increase of the number of theoretical plates, the increasing rate of the recovery yield of the component becomes slower as shown gradient change. When the number of theoretical plates is increased to a certain extent, the recovery yield of the component keeps a constant value of less than $100 \%$. No matter how we continue to increase the number of theoretical plates, there is always a limit loss of light components. The heavy component collected at column bottom has the similar curve type as shown curve $\varphi_{2}$ in Figure 1. Recovery yield curve of the transition fraction product at column top is curve $\lambda_{12}$ in Figure 1. If the number of theoretical plates is too small, then there will be more transition fraction product recovery yield than an appropriate number of theoretical plate. It can be seen that it is not beneficial when the number of theoretical plates is too high or too low discussing the products yield and the equipment investment as we understood before. 


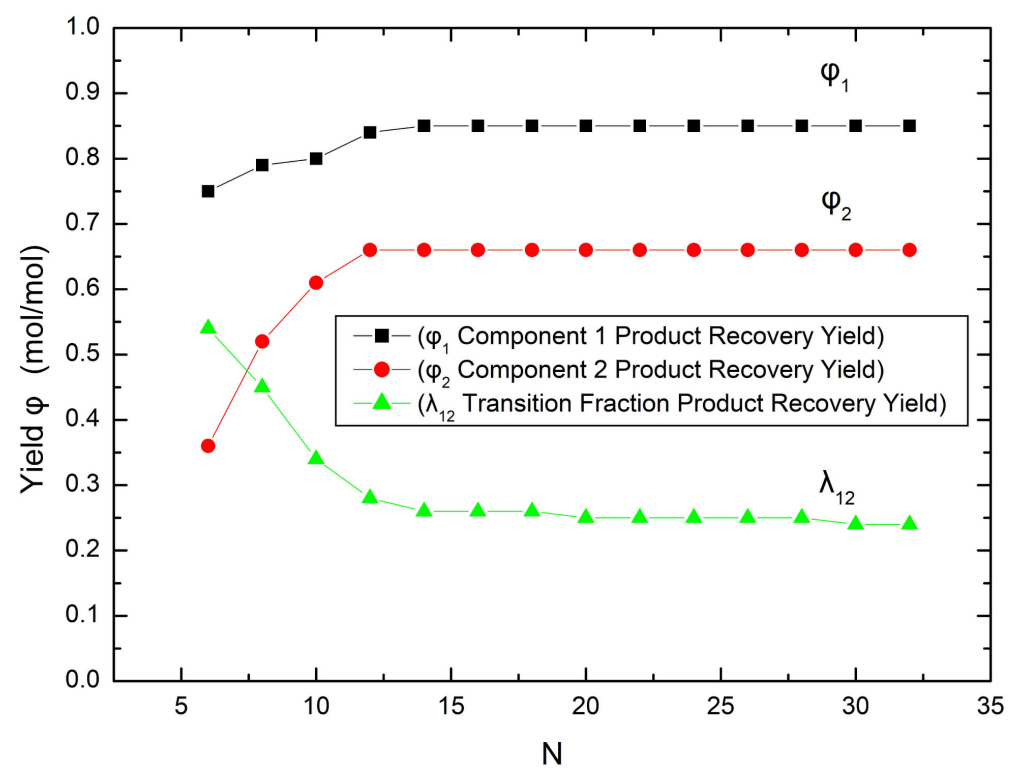

Figure 1. Schematic diagram to effect of the number of the theoretical plates on product recovery yield.

Therefore, there must exist an optimal number of theoretical plates when a batch distillation column is designed.

\subsubsection{Liquid Holdup at Column Top}

The liquid holdup at column top is an important parameter for batch distillation, especially for the external reflux distillation equipment. On the one hand, it has storage effect on liquid discharge. On the other hand, it is also conducive to the regulation of reflux ratio. When some fluctuations occur in the operation, the concentration at column top is able to be stabilized and liquid holdup at column top existence has a great influence on the separation effect. Figure 2 is a schematic diagram of the effects of liquid holdup on recovery yields of light component product, heavy component product and transition fraction product. It shows that the increase of liquid holding capacity at column top always decreases the recovery yield of the product, and that the recovery yield of the heavy component product is often lower than that of the light component product. Meanwhile the transition fraction product will increase gradually with the increase of the liquid holdup at column top. Therefore, the analysis shows that it is also important to select an appropriate liquid holdup at column top.

\subsubsection{Liquid Holdup at Column Body}

During the distillation process the "flywheel effect" is produced by the absorption or the release of volatile components with the increase or decrease of the concentration in the column. As an inertial effect, the "flywheel effect" makes the concentration change in the column slow, and encourages the trailing of the light component concentration. However, for the product distillation section that will be ended, high product concentration can be maintained at column top for a long time. Therefore, when the liquid holdup in column body is small, the 
increase of the liquid holdup in column body is beneficial to the increase of the recovery yields $\varphi_{1}$ and $\varphi_{2}$ of the light component product and the heavy component product as shown in Figure 3, but the recovery yields will decrease with the further increase of the liquid holdup of the column body.

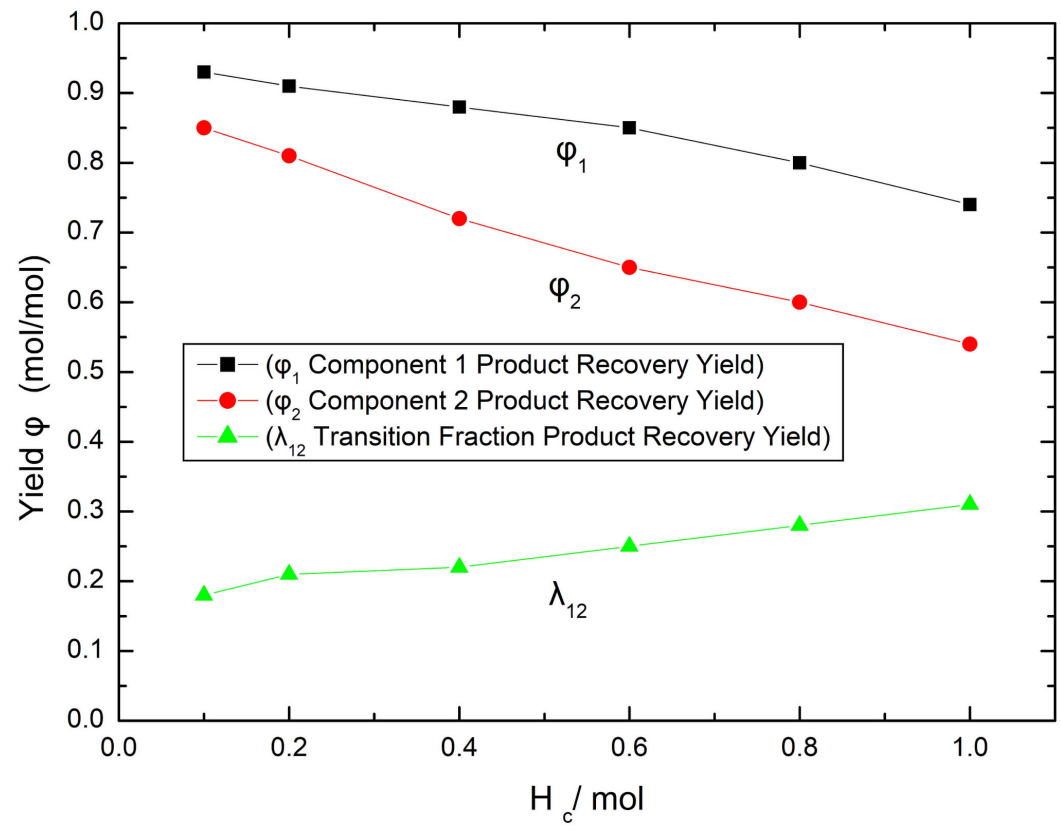

Figure 2. Schematic diagram to effect of the liquid holdup at column top on product recovery yield.

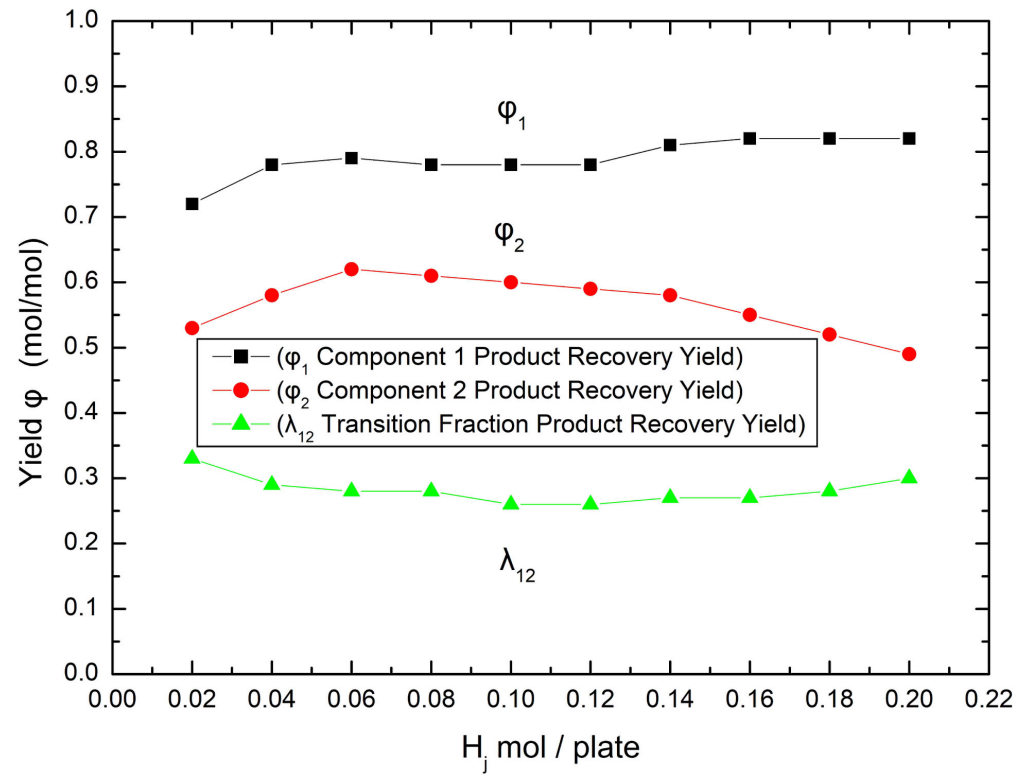

Figure 3. Schematic diagram to effect of the liquid holdup in column body on product recovery yield.

The increase of the liquid holdup in column body is beneficial to the increase of the recovery yields $\varphi_{1}$ and $\varphi_{2}$ of the light component product and the heavy 
component product as shown in Figure 3, but the recovery yields will decrease with the further increase of the liquid holdup of the column body. The decrease is mainly due to the recovery yield increase of non-product distillates, transition fraction products $\lambda_{12}$, with the increase of liquid holdup in the column, which affects the yields $\varphi_{1}$ and $\varphi_{2}$ of the light component product and the heavy component product. Therefore, it is also important to control the liquid holdup in column body reasonably.

\subsection{Maximal Yield and Limit Loss Proportion}

For a definite separation task which is completed on a fixed batch distillation column, the product recovery yield at column top is always less than $100 \%$ when the required concentration of the product at column top can't be maintained, there is always a residual light component in the column and they will be treated as a transition product. The loss proportion in this section refers to the fraction of the residual light component in the total feed quantity of the component, which is determined by the separation requirements and the equipment parameters of the column. For different operation strategies, the values of recovery yield and loss proportion are different. Due to the existence of liquid holdup in the column, there is a theoretical maximum yield and a limit loss proportion. Obviously, the sum of the maximum yield and the limit loss proportion is equal to one. An analytical expression for calculating limit loss proportion is derived by indefinitely prolonging the operation time of constant concentration at column top. For the operation of constant concentration at column top, the product output at column top tends to be a limit value with the increase of time, which is assumed to be $D_{y}$.

Thus, the limit loss proportion to the light component can be expressed as

$$
e=\frac{H_{B}^{0} x_{B}^{0}-D_{y} x_{D}}{H_{B}^{0} x_{B}^{0}}
$$

where $e, H_{B}^{0}, x_{B}^{0}$ and $x_{D}$ are the limit loss proportion, the total feed quantity, the raw material concentration and the product concentration, respectively.

Assume the liquid holdup at column top and the liquid holdup per plate in column body are $H_{c}$ and $H_{j}$ and let $\eta$ and $x_{B, \min }$ be average mole fraction of the light component in column body and mole fraction of the light component at column bottom. Thus, the Equation (2) and the Equation (3) can be drawn by mass balance of the light component and by total mass balance.

$$
\begin{gathered}
e H_{B}^{0} x_{B}^{0}=H_{B} x_{B}+H_{j} N \eta+H_{c} x_{D} \\
H_{B}=H_{B}^{0}-D_{y}-H_{j} N-H_{c}
\end{gathered}
$$

Combining the Equation (2) and the Equation (3) output of the light component distillate product can be calculated from the Equation (4).

$$
D_{y}=H_{B}^{0} x_{B}^{0}(1-e) / x_{D}
$$

The limit of constant concentration at column top must tend to total reflux 
operation and thus the minimum value of the concentration at column bottom, $x_{B, \min }$, can be obtained from the Fenske equation as shown the Equation (5).

$$
x_{B, \min }=\frac{x_{D}}{\alpha^{N-1}-x_{D}\left(\alpha^{N-1}-1\right)}
$$

where $N$ is the number of the theoretical plate.

It is known from Fenske equation that the concentration of the light component on the first, the second and even the $n^{\text {th }}$ plate can be obtained from the following the Equation (6)

$$
x_{n}=\frac{x_{D}}{\alpha^{n}\left(1-x_{D}\right)+x_{D}}
$$

where $n$ is the code of the theoretical plate.

Thus, the average concentration of the light component in column body is

$$
\eta=\frac{1}{N} \int_{1}^{N} x_{n} \mathrm{~d} n=1-\frac{1}{N \ln \alpha} \ln \frac{\left[\left(1-x_{D}\right) \alpha^{N}+x_{D}\right] \alpha}{\left(1-x_{D}\right) \alpha+x_{D}}
$$

where $\eta$ is average mole fraction of light component in column body.

Further, the limit loss proportion $e$ can be calculated by the Equation (8) by combining the Equation (2), the Equation (3), the Equation (4) and the Equation (5).

$$
e=e_{1}+e_{2}+e_{3}
$$

where the three sub-items $e_{1}, e_{2}$ and $e_{3}$ are expressed as follows

$$
\begin{gathered}
e_{1}=\frac{x_{D}-x_{B}^{0}}{x_{B}^{0}\left(\alpha^{N+1}-1\right)\left(1-x_{D}\right)} \\
e_{2}=\frac{N H_{j}}{H_{B}^{0} x_{B}^{0}}\left[\eta-\frac{x_{D}-\eta}{\left(1-x_{D}\right)\left(\alpha^{N+1}-1\right)}\right] \\
e_{3}=\frac{H_{c} x_{D}}{H_{B}^{0} x_{B}^{0}} .
\end{gathered}
$$

From the mathematical expression of the limit loss proportion, it can be seen that the value of limit loss proportion is determined by the physical properties, separation requirements and equipment parameters of the separated object system for a specific separation task and the value is independent of the operating parameters. In Figure 4, the change relations between the limit loss proportion and the number of the theoretical plates are described at fixed relative volatility $1.5,2,2.5$ and 3 . It can be seen that the limit loss proportion is greatly affected when the number of theoretical plates $N$ is smaller and that the decrease of the limit loss proportion value is extremely fast. Increasing number of the theoretical plates is beneficial to increase the recovery yield of the light component. When the number of the theoretical plates continue to turn larger, there is no obvious change in value of the limit loss proportion with the increase of the number of theoretical plates. In other words, excessive increase in the number of the theoretical plate does not have much effect on the recovery yield increase of the light 
component product, but it will only make the investment increase in equipment. Therefore, choosing an appropriate number of theoretical plates not only can reduce the investment of equipment, but also higher product recovery yield can be reached, which will be extraordinary instructive to the design of batch distillation column.

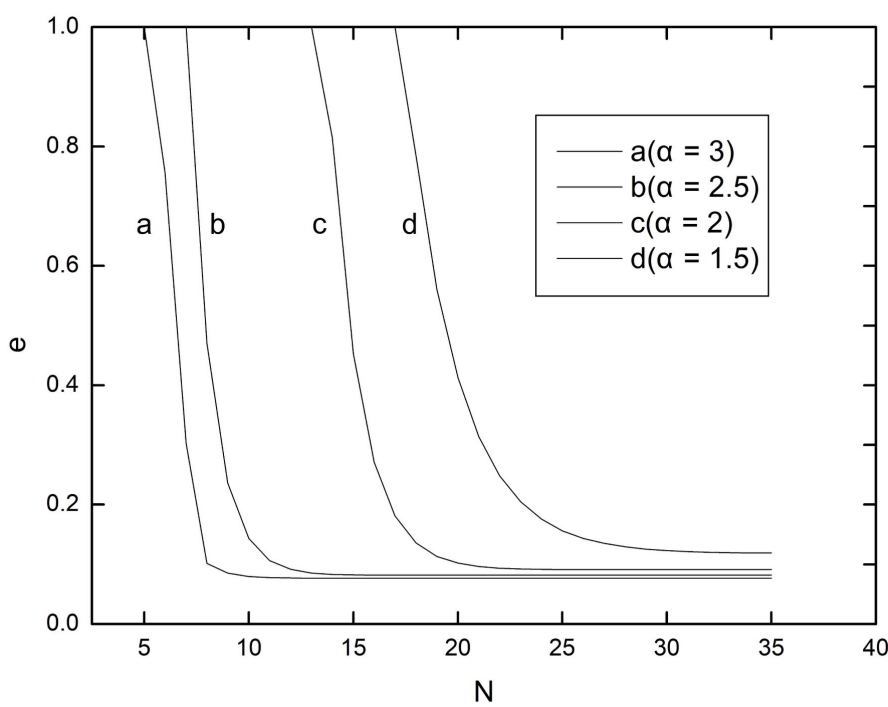

Figure 4. Schematic diagram to effect of the number of the theoretical plates on the limit loss proportion $\left(x_{B}^{0}=0.4, \eta=0.7, x_{D}=0.999, H_{c} / H_{B}^{0}=2.26 \%, H_{i} / H_{B}^{0}=0.154 \%\right)$.

Although the limit loss proportion is derived from the binary component liquid mixture separation system, it is also applicable to the batch distillation process of the multi-component separation system. In this case, the product recovery yield of the multi-component batch distillation is further reduced by the effect of the trailing of the former component. But it hardly can affect the use of the approach of the limit loss proportion in the batch distillation design because the multi-component system can be simplified to become numerous binary adjacent component system. Therefore, the influence curve of the theoretical plate number on the limit loss proportion still can have guided the design of the batch distillation column.

\section{Separation Difficulty and Redundancy Coefficient}

\subsection{Definition of Separation Difficulty}

By discussing the effect of operation parameters on the different component product recovery yield for a certain type batch distillation column, the main operating parameters that affect the recovery yield of the component product herein can be correlated into a new factor to simplify the design of the batch distillation design, which is called separation difficulty. Assume that a binary liquid mixture with an average relative volatility $\alpha$ is separated from an initial state $x_{B}^{0}$ to a final state $x_{D}$ under the separation action of a batch distillation column. Considering Fenske equation expression and easy use the mathematical 
expression of the separation difficulty $\beta$ employed is defined as:

$$
\beta=\lg \left[\left(\frac{x_{D}}{1-x_{D}}\right)\left(\frac{1-x_{B}^{0}}{x_{B}^{0}}\right)\right] / \alpha
$$

where $\beta, x_{D}, x_{B 0}$ and $\alpha$ respectively are separation difficulty, mole fraction of the light component at column top, the initial mole fraction of the light component at column bottom and the average relative volatility of the light component relative to the heavy component.

\subsection{Redundancy Coefficient Curve}

For the task of a certain separating difficulty, the critical theoretical plate number $N_{c}$ is obtained from the limit loss proportion approach. In order to keep a certain margin, a redundancy coefficient $\zeta$ is given. Thus, the number of the theoretical plates determined is

$$
N=\zeta N_{c}
$$

where $N, N_{c}$ and $\zeta$ are the actual number of the theoretical plates, the critical theoretical plate number and the redundancy coefficient, respectively.

Take different $\zeta$ values, a series of theoretical plates can be gotten. Afterwards, the minimum number of theoretical plates, which have little effect on the recovery yield increase of the component product by the algorithm [29] [30], is determined. At this time the $\zeta$ value is the redundancy coefficient at this difficulty. Using different separation difficulties, a series of $\zeta$ values can be gotten.

Taking the separation difficulty $\beta$ as the horizontal coordinate and the redundancy coefficient $\zeta$ as the longitudinal ordinate, the relation curve of separation difficulty $\beta$ and redundancy coefficient $\zeta$ is drawn as shown in Figure 5.

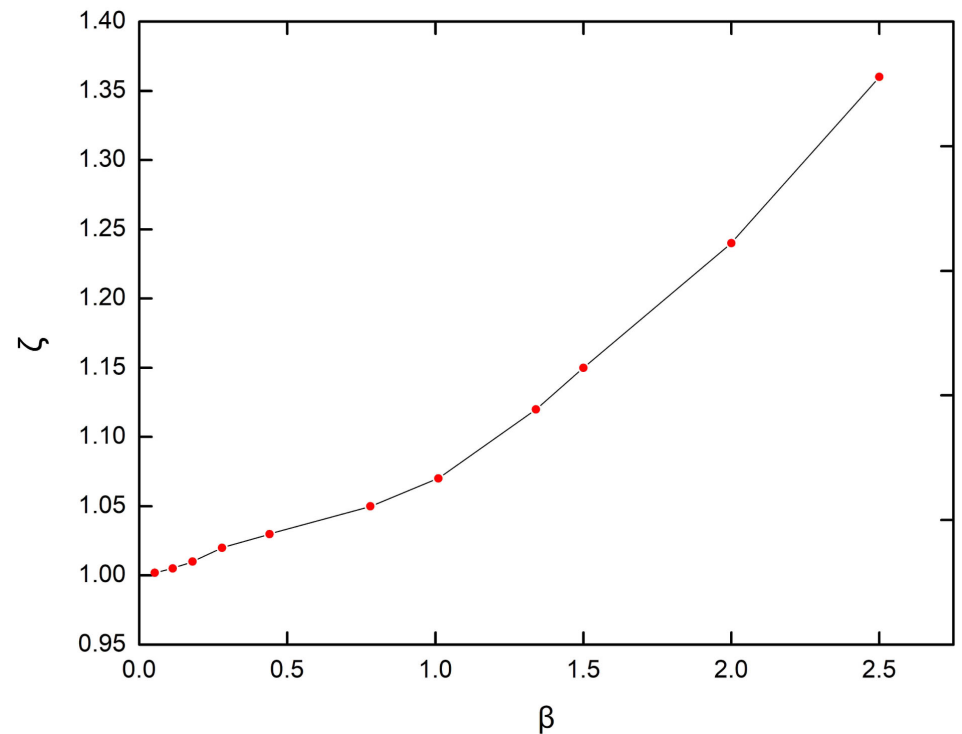

Figure 5. Schematic diagram of relation between separation difficulty and redundancy coefficient $[\beta \in(0,2.5)]$. 


\section{Determination of the Number of Theoretical Plates}

For a separation task with a certain separation difficulty, the minimum number of theoretical plates is given according to the relationship between the limit loss proportion and the number of theoretical plates. And then the actual number of theoretical plates in batch distillation column is determined by the correction of the redundancy coefficient under the assistance of the separation difficulty.

The specific steps are shown as follows:

1) Select column diameter and packing type according to the requirement of processing capacity and estimate the liquid holdup each plate in column body $H_{j}$ and the liquid holdup at column top $H_{c}$.

2) Draw the relation curve of $N \sim e$ according to physical properties data of matter and separation requirements.

3) Find the critical number value of theoretical plates between the $N \sim e$ sensitive area and the $N \sim e$ flat area in the $N \sim e$ relation curve.

4) Start to separate the next component taking the stock in the column bottom as a raw material, and the material quantity and the concentration of the components in the column bottom are calculated by material balance.

5) Repeat the steps 2, 3 and 4 until the required product indicators at column top are fully calculated.

6) Compare $N_{c}$ values for each component group and take the maximum critical number of theoretical plates $N_{c}^{\prime}$ to become the minimum number of theoretical plates required.

7) See Figure 5 and take the redundancy coefficient under this separation difficulty, the final number of theoretical plates determined can be expressed as

$$
N=\zeta N_{c}^{\prime}
$$

\section{Example to Calculation of Column Height of Batch Distillation}

By using following steps, the height of batch distillation column can be determined.

1) Identify design task. A raw liquid mixtures that are composed of ethanol, $n$-propanol and $n$-butanol with mass fraction ratio $0.4,0.2$ and 0.4 need to be separated by a batch distillation column. Calculate the column height that can make product concentration required of ethanol and $n$-propanol be 99 percent and 78 percent at column top and that can process 2.5 ton raw material per batch. Assume that there are two operational periods to the distilled products at column top. The first operational period refers to the time range that the first component product is being distilled off until the beginning of the first transition fraction product is being distilled off at column top. The second operational period refers to the time range that the second component is being distilled off until the beginning of the second transition fraction product is being distilled off at column top.

2) Select the BX type wire mesh corrugated packing with the column diameter 
$0.6 \mathrm{~m}$. The height of equal plate $\varepsilon$ is $0.3 \mathrm{~m}$. The liquid holdup in packing is $5 \%$ of the volume of packing layer. By accounting, the liquid holdup each plate in column body $H_{j}$ is $3.4 \mathrm{~kg}$ and the liquid holdup $H_{c}$ at column top is $50 \mathrm{~kg}$.

3) Plot the $N \sim e$ relation curve in the first operation period that is from the first component product distilling off to the beginning of the transition fraction product distilling off at column top. Take common relative volatility between ethanol and $n$-propanol 2.08 as the value calculated. The curve a in Figure 6 can be drawn from the Equation (7), Equation (8) and the used data in Figure 4.

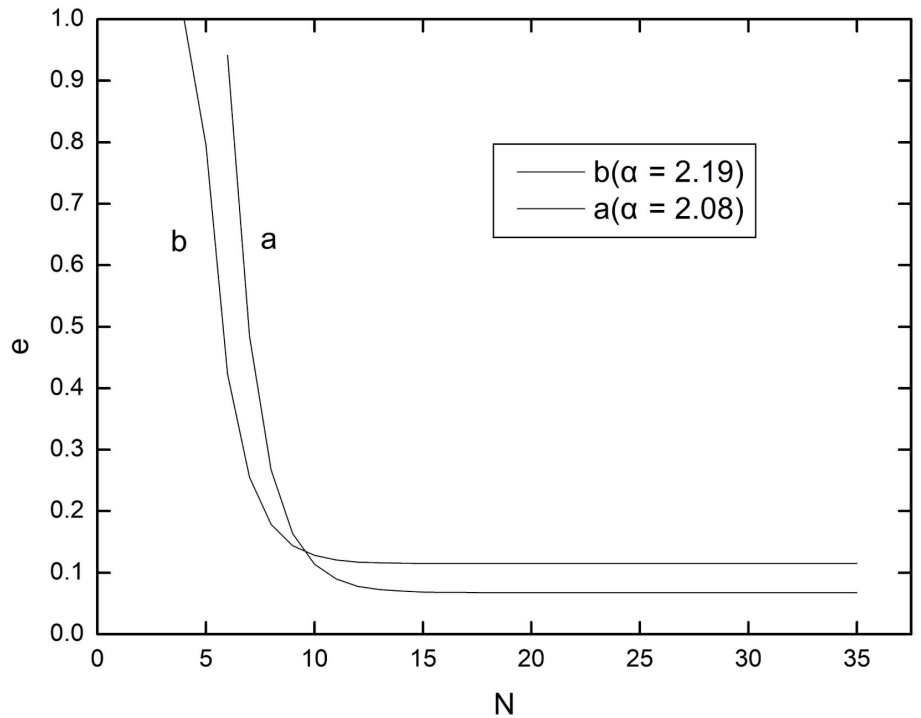

Figure 6. Schematic diagram of relation of number of theoretical plate and limit loss proportion $\left(x_{B}^{0}=0.4, \eta=0.7, x_{D}=0.99, H_{c} / H_{B}^{0}=2.26 \%, H_{j} / H_{B}^{0}=0.154 \%\right)$.

4) Get critical number of the theoretical plate. See Figure 6. The critical number of the theoretical plate $N_{c_{1}}$ is 15 and the limit loss proportion $e$ is 0.0681 .

5) Find the initial conditions of the second operating period. The maximum yield of ethanol product derived from the material balance is $D_{y}=\frac{H_{B}^{0} x_{B}^{0}(1-e)}{x_{D_{1}}}$, whose result is $941 \mathrm{~kg}$.

Assume the amounts of transition fraction products are close to the sum of the liquid holdup at column top and the liquid holdup in column body, the ethanol mass lost in the first component product and the transition fraction product is

$$
S_{2}=\left[\left(H_{c}+H_{j} N_{c_{1}}\right)-H_{B}^{0} x_{B_{1}}^{0} e\right]-D_{y}\left(1-x_{D_{1}}\right),
$$

whose value is $30.3 \mathrm{~kg}$ by calculation.

Furthermore, the mass of solutions at column bottom is

$$
H_{B}=\left(H_{B}^{0} x_{B_{2}}^{0}-S_{2}\right)+H_{B_{3}}^{0} x_{B_{3}}^{0},
$$

whose value calculated is $1469.7 \mathrm{~kg}$. Simultaneously concentrations of $n$-propanol and $n$-butanol calculated at column bottom are $x_{B_{2}}=0.3196$, and $x_{B_{3}}=0.6804$, 
respectively.

6) Calculate the number value of theoretical plates required for the second operational period. Take common relative volatility between $n$-propanol and $n$-butanol 2.19 as a calculated value. The curve b in Figure 6 can be drawn from the Equation (7), Equation (8) and from those data used in Figure 4. Afterwards, see Figure 6. The results are that the critical number of the theoretical plate $N_{c_{2}}$ is 12 and the limit loss proportion $e$ is 0.1158 , respectively.

7) Compare their sizes for the critical numbers of the theoretical plate $N_{c_{1}}$ and $N_{c_{2}}$. Since $N_{c_{1}}$ is greater than $N_{c_{2}}$, then take the practical critical theoretical plate number $N_{c}^{\prime}=N_{c_{1}}=15$. Moreover, the separation difficulty in the system is $\beta=\lg \left[\left(\frac{x_{D_{1}}}{1-x_{D_{1}}}\right)\left(\frac{1-x_{B_{1}}^{0}}{x_{B_{1}}^{0}}\right)\right] / \alpha$, whose value is 1.044 after the verification is completed. See Figure 5, the redundancy coefficient $\zeta=1.08$. Therefore, the actual number of theoretical plates can be calculated as follows

$$
N=\zeta N_{c}^{\prime}=1.08 \times 15=16.2
$$

The actual number values of theoretical plates are rounded to become $N=17$, then the height of the batch distillation column can be drawn from the below.

$$
Z=\varepsilon N=0.3 \times 17=5.1 \mathrm{~m}
$$

Finally, the height of the batch distillation is $5.1 \mathrm{~m}$.

\section{Conclusions}

By discussing the effects of equipment parameters such as the number of the theoretical plate, the liquid holdup at column top and the liquid holdup at column body in a batch distillation column on maximal yields of light component and of heavy component in a binary component separation system, change curves of the limit loss proportion of the light component and of the heavy component with the numbers of theoretical plate are built. The curves can become a requisite in this design tactic of a batch distillation column and can be used to guide equipment selection of batch separation system of liquid mixture of whether simple binary component or complex multi-component by using following procedures:

1) Compute the values of separation difficulty defined according to the known data between adjacent components in binary component or multi-component system.

2) Identify the value of redundancy coefficient in terms of the steps said in the design technique including the sample mentioned.

3) Get the actual number of theoretical plates and reduce the height of the batch distillation column.

4) In alcohol mixture separation system of the sample given, the actual number of theoretical plates and the height of batch distillation column with column diameter $0.6 \mathrm{~m}$ are 17 and $5.1 \mathrm{~m}$, respectively. 
To sum up, the design approach of the batch distillation column described in this paper is simple and feasible as shown in computational sample and it also will be able to be used to design multi-component batch distillation column when the adjacent components are regarded as numerous binary components. Moreover, some investigations such as a unity of separation difficulty to different systems needed to be separated; the augmentation of curve with regard to separation difficulty and redundancy coefficient for designing batch distillation column, novel operational control strategy and modified optimization algorithm in batch distillation simulation will become future investigation target after this work and synchronously novel research hotspots will come into being.

\section{Acknowledgements}

The financial support from the United Laboratory Construction Fund of China and of Liaoning Province (No. 2011), the Natural Science Foundation of Liaoning Province (No. 2013020150), the Program for Liaoning Excellent Talents in University (No. LJQ2011134), and the Science and Technology Fund from Shenyang Institute of Engineering (No. XNZD-1807) are gratefully acknowledged.

\section{Conflicts of Interest}

The author declares no conflicts of interest regarding the publication of this paper.

\section{References}

[1] Rodriguez-Donis, I., Gerbaud, V., Lavoine, S., Meyer, M., Thiebaud-Roux, S. and Dupouyet, A. (2019) Modelling and Experimental Validation of Dimethyl Carbonate Solvent Recovery from an Aroma Mixture by Batch Distillation. Chemical Engineering Research and Design, 147, 1-17. https://doi.org/10.1016/j.cherd.2019.04.007

[2] Haigh, K.F., Petersen, A.M., Gottumukkala, L., Mandegari, M., Naleli, K. and Görgens, J.F. (2018) Simulation and Comparison of Processes for Biobutanol Production from Lignocellulose via ABE Fermentation. Biofuels, Bioproducts and Biorefining, 12, 1023-1036. https://doi.org/10.1002/bbb.1917

[3] Hegely, L. and Lang, P. (2018) Optimization of Batch Heteroazeotropic Distillation Operational Strategies with Entrainer Recycle. Computer Aided Chemical Engineering, 43, 1505-1511. https://doi.org/10.1002/ep.12872

[4] Marquez-Ruiz, A., Ludlage, J.H.A. and Özkan, L. (2018) Optimization and Low-Level Control Design for Reactive Batch Distillation Columns Including the Start-Up. Computer Aided Chemical Engineering, 44, 577-582.

https://doi.org/10.1016/B978-0-444-64241-7.50091-4

[5] Arellano-Garcia, H., Carmona, I. and Wozny, G. (2008) A New Operation Mode for Reactive Batch Distillation in Middle-Vessel Columns: Start-Up and Operation. Computers and Chemical Engineering, 32, 161-169.

https://doi.org/10.1016/j.compchemeng.2007.08.002

[6] Leipold, M., Gruetzmann, S. and Fieg, G. (2009) An Evolutionary Approach for Multi-Objective Dynamic Optimization. Computers and Chemical Engineering, 33, 
857-870. https://doi.org/10.1016/j.compchemeng.2008.12.010

[7] Fanaei, M.A., Dehghani, H. and Nadi, S. (2012) Comparing and Controlling of Three Batch Distillation Column Configurations for Separating Tertiary Zeotropic Mixtures. Scientia Iranica, 19, 1672-1681. https://doi.org/10.1016/j.scient.2012.09.005

[8] Luyben, W.L. (2015) Aspen Dynamics Simulation of a Middle-Vessel Batch Distillation Process. Journal of Process Control, 33, 49-59. https://doi.org/10.1016/j.jprocont.2015.06.002

[9] Zhu, Z., Li, X., Cao, Y., Liu, X. and Wang, Y. (2016) Design and Control of a Middle Vessel Batch Distillation Process for Separating the Methyl Formate Methanol Water Ternary System. Industrial \& Engineering Chemistry Research, 55, 2760-2768. https://doi.org/10.1021/acs.iecr.5b04067

[10] Zhu, M., Hou, Y., Yu, N., Chen, M., Ma, Z. and Sun, L. (2018) Design and Control of a Middle-Vessel Batch Distillation for Separating DMC-EMC-DEC Mixture. Chinese Journal of Chemical Engineering, 26, 1837-1844. https://doi.org/10.1016/j.cjche.2017.11.014

[11] Demicoli, D. and Stichlmair, J. (2004) Separation of Ternary Mixtures in a Batch Distillation Column with Side Withdrawal. Computers and Chemical Engineering, 28, 643-650. https://doi.org/10.1016/j.compchemeng.2004.02.009

[12] Gruetzmann, S., Fieg, G. and Kapala, T. (2006) Theoretical Analysis and Operating Behaviour of a Middle Vessel Batch Distillation with Cyclic Operation. Chemical Engineering and Processing, 45, 46-54. https://doi.org/10.1016/j.cep.2005.05.005

[13] Parhi, S.S., Rangaiah, G.P. and Jana, A.K. (2019) Optimizing Reboiler Duty and Reflux Ratio Profiles of Vapor Recompressed Batch Distillation. Separation and Purification Technology, 213, 553-570. https://doi.org/10.1016/j.seppur.2018.12.066

[14] Jana, A.K. and Sinha, R. (2012) Comparative Control Study of a Simulated Batch Rectifier. Computers and Chemical Engineering, 36, 265-272. https://doi.org/10.1016/j.compchemeng.2011.09.013

[15] Prasad, V. and Bequette, B.W. (2003) Nonlinear System Identification and Model Reduction Using Artificial Neural Networks. Computers and Chemical Engineering, 27, 1741-1754. https://doi.org/10.1016/S0098-1354(03)00137-6

[16] Fileti, A.M.F., Antunes, A.J.B., Silva, F.V., Silveira, V. and Pereira, J.A.F.R. (2007) Experimental Investigations on Fuzzy Logic for Process Control. Control Engineering Practice, 15, 1149-1160. https://doi.org/10.1016/j.conengprac.2007.01.009

[17] Kao, Y.L. and Ward, J.D. (2016) Simultaneous Optimization of the Design and Operation of Batch Reactive Distillation Processes. Industrial and Engineering Chemistry Research, 55, 267-278. https://doi.org/10.1021/acs.iecr.5b03170

[18] Diwekar, U.M. and Madhavan, K.P. (1991) Multicomponent Batch Distillation Column Design. Industrial and Engineering Chemistry Research, 30, 713-721. https://doi.org/10.1021/ie00052a014

[19] Diwekar, U.M. (1991) An Efficient Design Method for Binary, Azeotropic, Batch Distillation Columns. AIChE Journal, 37, 1571-1578. https://doi.org/10.1002/aic.690371014

[20] Bernot, C., Doherty, M.F. and Malone, M.F. (1993) Design and Operating Targets for Nonideal Multicomponent Batch Distillation. Industrial and Engineering Chemistry Research, 32, 293-301. https://doi.org/10.1021/ie00014a008

[21] Salomone, H.E., Chiotti, O.J. and Iribarren, O.A. (1997) Short-Cut Design Proce- 
dure for Batch Distillations. Industrial and Engineering Chemistry Research, 36, 130-136. https://doi.org/10.1021/ie950458n

[22] Sharif, M., Shah, N. and Pantelides, C.C. (1998) On the Design of Multicomponent Batch Distillation Columns. Computers and Chemical Engineering, 22, S69-S76. https://doi.org/10.1016/S0098-1354(98)00040-4

[23] Low, K.H. and Sørensen, E. (2003) Simultaneous Optimal Design and Operation of Multivessel Batch Distillation. AICHE Journal, 49, 2564-2576. https://doi.org/10.1002/aic.690491011

[24] Huerta-Garrido, M.E., Rico-Ramírez V. and HernÃndez-Castro, S. (2004) Simplified Design of Batch Reactive Distillation Columns. Industrial and Engineering Chemistry Research, 43, 4000-4011. https://doi.org/10.1021/ie030658w

[25] Espinosa, J., Salomone, E. and Iribarren, O.A. (2004) Computer-Aided Conceptual Design of Batch Distillation Systems. Industrial and Engineering Chemistry Research, 43, 1723-1733. https://doi.org/10.1021/ie0302589

[26] Narvaez-Garcia, A., Zavala-Loria, J.D.C., Vilchis-Bravo, L.E. and Rocha-Uribe, J.A. (2013) Design of Batch Distillation Columns Using Short-Cut Method at Constant Reflux. Journal of Engineering, 2013, Article ID: 685969.

https://doi.org/10.1155/2013/685969

[27] Swaminathan, J. and Lienhard, J.H.V. (2018) Design and Operation of Membrane Distillation with Feed Recirculation for High Recovery Brine Concentration. Desalination, 445, 51-62. https://doi.org/10.1016/j.desal.2018.07.018

[28] Hao, W.F. (2002) Study on Simulation and Optimal Design of Multi-Component Batch Distillation. Master's Degree Dissertation of Shenyang Institute of Chemical Technology, Shenyang.

[29] Hao, W.F., Fan, S.S., Li, W.X., Wan, J.Q. and Yin, D.H. (2004) Simulation and Experiment of Three Component Alcohol Mixtures in Batch Distillation. Journal of Chemical Industry and Engineering, 55, 934-941.

[30] Gerbaud, V., Rodriguez Donis, I., Hégely, L., Lâng, P., Denes, F. and You, X. (2019) Review of Extractive Distillation. Process Design, Operation, Optimization and Control. Chemical Engineering Research and Design, 141, 229-271. https://doi.org/10.1016/j.cherd.2018.09.020 


\section{Nomenclature}

$B$ : Column bottom (-)

c. Column top (-)

$D$ : Output of light component distillate product $(\mathrm{kg})$

$D$ : Distillate product (-)

e. Limit loss proportion (-)

$H$ : Initial feeding quantity $(\mathrm{mol})$

$H$ : Liquid holdup at column top, column plate, column body and column bottom (mol or $\mathrm{kg}$ )

$N$ : Number of the theoretical plate (-)

$n$ : Code of the theoretical plate (-)

$S$ : Ethanol mass lost in the first component product and the transition fraction product $(\mathrm{kg})$

$x$ : Mole fraction of different components raw material and mole fraction of the light component, heavy component and transition fraction product at column top, on column plate, in column body and at column bottom (-)

$y$. Yield amount expression (-)

$Z$ : Actual height of the batch distillation column (m)

\section{Special characters}

$\alpha$ : Average relative volatility of the light component relative to the heavy component (-)

$\beta$ : Separation difficulty of the separation system (-)

$\eta$ : Average mole fraction of the light component in column body (-)

$\varepsilon$. Height of equal plate $(\mathrm{m})$

$\zeta$ : Redundancy coefficient (-)

$\varphi$ : Recovery yield of the component product ( $\mathrm{mol} / \mathrm{mol}$ )

$\lambda$ : Recovery yield of the transition fraction product ( $\mathrm{mol} / \mathrm{mol}$ )

\section{Superscript}

0: Initial State

\section{Subscript}

1, 2, 3: Component, numerical code or operational period

c. Critical value or column top

i: Column body

j: Column plate

min: Minimum value 